\title{
Recent range-wide demographic expansion in a Taiwan endemic montane bird, Steere's Liocichla (Liocichla steerii)
}

\author{
Bailey D McKay ${ }^{1,2^{*}}$, Herman L Mays $\mathrm{Jr}^{3}$, Yi-Wen Peng ${ }^{4}$, Kenneth H Kozak ${ }^{1,5}$, Cheng-Te Yao ${ }^{6,7}$, Hsiao-Wei Yuan ${ }^{4}$
}

\begin{abstract}
Background: The subtropical island of Taiwan is an area of high endemism and a complex topographic environment. Phylogeographic studies indicate that vicariance caused by Taiwan's mountains has subdivided many taxa into genetic phylogroups. We used mitochondrial DNA sequences and nuclear microsatellites to test whether the evolutionary history of an endemic montane bird, Steere's Liocichla (Liocichla steerii), fit the general vicariant paradigm for a montane organism.

Results: We found that while mountains appear to channel gene flow they are not a significant barrier for Steere's Liocichla. Recent demographic expansion was evident, and genetic diversity was relatively high across the island, suggesting expansion from multiple areas rather than a few isolated refugia. Ecological niche modeling corroborated the molecular results and suggested that populations of Steere's Liocichla are connected by climatically suitable habitat and that there was less suitable habitat during the Last Glacial Maximum.

Conclusions: Genetic and ecological niche modeling data corroborate a single history-Steere's Liocichla was at lower density during the Last Glacial Maximum and has subsequently expanded in population density. We suggest that such a range-wide density expansion might be an overlooked cause for the genetic patterns of demographic expansion that are regularly reported. We find significant differences among some populations in $F_{S T}$ indices and an admixture analysis. Though both of these results are often used to suggest conservation action, we affirm that statistically significant results are not necessarily biologically meaningful and we urge caution when interpreting highly polymorphic data such as microsatellites.
\end{abstract}

\section{Background}

Past climatic cycles have had a profound impact on the levels and distribution of genetic diversity [1]. Despite the fact that most biodiversity occurs in tropical areas, most studies have focused on the temperate regions in North America and Europe where the retreat of ice sheets has left widespread genetic patterns consistent with northward expansion from southern refugia [2]. This bias has left us with a poorer understanding of the impact of climate cycles on the phylogeographical patterns of tropical taxa. However, initial phylogeographic studies indicate that tropical areas have been impacted by climatic cycles and that tropical species can have deep intraspecific lineages with complex histories [2,3].

\footnotetext{
* Correspondence: mckay117@umn.edu
'Bell Museum of Natural History, University of Minnesota, St Paul, MN 55108,

* Correspondence: mckay117@umn.edu
'Bell Museum of Natural History, University of Minnesota, St Paul, MN 55108, USA
}

(c) 2010 McKay et al; licensee BioMed Central Ltd. This is an Open Access article distributed under the terms of the Creative Commons Attribution License (http://creativecommons.org/licenses/by/2.0), which permits unrestricted use, distribution, and reproduction in any medium, provided the original work is properly cited. The subtropical island of Taiwan is an area of high diversity. Taiwan first emerged from the sea following the collision of the Eurasian and Philippine Sea plates approximately 5 mya [4,5]. Despite periodic connections with the mainland during low sea levels [6], Taiwan's general isolation from mainland Asia has given rise to many endemic species and subspecies. Within the island, mountains have provided additional opportunities for isolation. This is reflected in the phylogeographic patterns found in some lowland taxa. For example, a freshwater crab (Candidiopotamon rathbunae) [7], a frog (Fejervarya limnocharis) [8], and a gecko (Gekko hokouensis) [9] exhibit a common pattern of genetic division east and west of the Central Mountain Range (CMR), the prominent mountain range that runs through much of the longitudinal length of the island 
(Fig. 1). In contrast, many montane species show a pattern of north-south genetic division: e.g. Formosan Wood Mouse (Apodemus semotus) [10], a ranid frog (Rana sauteri) [11], and Siberian Weasel (Mustela sibirica) [12]. The observation of common phylogeographic patterns in such varied taxa suggests vicariance as the cause [13].

The east-west pattern of genetic division has been found in lowland birds (i.e. Light-vented and Taiwan Bulbul Pycnonotus sinensis and P. taivanus, [14]; Ringnecked Pheasant Phasianus colchicus, [15], however, no montane bird has yet been phylogeographically surveyed. Determining whether montane birds were affected by the same isolating events that generated genetic divisions in other montane taxa is important for understanding the impact of mountain vicariance in Taiwan. In a conservation context, uncovering historical lineages is essential for guiding management efforts, especially for species confined to single islands. Steere's Liocichla (Liocichla steerii) is a montane, resident bird species endemic to Taiwan. It inhabits forest edge and secondary growth from approximately $1000 \mathrm{~m}$ to 3000 $\mathrm{m}$. Its dispersal ability was inferred to be low [16], and high song diversity also suggests dispersal may be low [17]. Therefore, Steere's Liocichla is an ideal avian candidate to have developed the same genetic structure observed in potentially less vagile vertebrates.

In this paper, we used mitochondrial DNA sequences and nuclear microsatellites to investigate the evolutionary history of Steere's Liocichla. Our primary goal was to determine whether this endemic species showed the same phylogeographic patterns of deep divergence that characterize other montane vertebrates in Taiwan. As a supplement to our genetic data, we used ecological niche modeling to predict this species' range during the Last Glacial Maximum (LGM). We also considered whether any populations of Steere's Liocichla might be of special management concern for conservation efforts.

\section{Methods}

\section{Sampling and laboratory methods}

A total of 122 individuals was sampled from 13 populations located throughout the breeding range of the species (Fig. 1; Table 1). Birds were caught during the breeding season (March to September) by mist net and $50 \mu \mathrm{l}$ of blood was collected via brachial venipuncture. Blood samples were stored in lysis buffer [18] at room temperature until they could be transported to the laboratory where they were stored at $-20^{\circ} \mathrm{C}$.

Whole genomic DNA was extracted using a standard phenol-chloroform protocol followed by ethanol precipitation. The mitochondrial NADH dehydrogenase subunit 2 (ND2) gene was amplified from a subset of 91 individuals representing all populations (Table 1) using the primers L5216 and H6313 [19]. Polymerase chain reaction (PCR) was performed in $10 \mu \mathrm{l}$ reactions on a MJ Research PTC-100 thermocycler using a thermal profile of $94^{\circ} \mathrm{C}$ for $4 \mathrm{~min}$ followed by 30 cycles of $1 \mathrm{~min}$ at $94^{\circ} \mathrm{C}, 1 \mathrm{~min}$ at $55^{\circ} \mathrm{C}$, and $2 \mathrm{~min}$ at $72^{\circ} \mathrm{C}$, and then 10 min at $72^{\circ} \mathrm{C}$. Primers and excess dNTPs were removed from the PCR product with ExoSAP-IT (USB Corporation) following the manufacturer's instructions. Sequencing was performed on an ABI 3700 automated sequencer using BigDye kit v. 3.0 according to recommended protocols (Applied Biosystems). All products were sequenced in both directions. Usually, the amplification primers were sufficient to obtain $>1,000$ base pairs (bp) of unambiguous sequence. However, whenever an ambiguous stretch of sequence was produced, internal sequencing primers (L5758 and H5766; [19]) were used to clarify ambiguous bases. Complementary strands were aligned and edited using SEQUENCHER v. 4.6 (GeneCodes Corporation). All sequences were inspected individually using the raw spectrograph data and every point mutation was checked for authenticity. Sequences have been deposited in GenBank (GU560065-GU560155).

All individuals were also genotyped at seven microsatellite loci. Four loci were developed specifically for Steere's Liocichla (lsgata17, lsgata 21, lsgata 24, lsgata 25; [20]) and three additional loci (GC-GATA10, GCGATA 15, GC-GATA 23; [21]) were originally developed for another babbler, the Hwamei (Garrulax canorus). Forward primers were fluorescently labeled, and the PCR cycling protocol followed the above for all loci except GC-GATA 15, for which we used an annealing temperature of $50^{\circ} \mathrm{C}$. Fragment analysis of the amplification product was conducted on a Beckman CEQ 8000 sequencer (Beckman Coulter).

\section{Genetic data analysis}

For mtDNA sequences, we estimated nucleotide diversity $(\pi)$, number of haplotypes (nh), and haplotype diversity (h) for each population using the program DnaSP $v$. 4.9 [22]. For each population, we also computed Fu's $F_{S}$, a neutrality statistic that is particularly sensitive to population demographic expansion [23]. Its significance was tested with 10,000 coalescent simulations using the program ARLEQUIN 3.11 [24]. We used a mismatch distribution analysis to estimate parameters of a demographic expansion using a generalized least-square approach [25], as implemented in ARLEQUIN. To estimate the time of expansion, we used the formula $\tau=2$ $\mu t$ [26], where $\tau$ is the time since the demographic expansion, $t$ is the number of generations, and $\mu$ is the mutation rate. We obtained a $95 \%$ confidence interval (CI) for $\tau$ with 1000 replicates of bootstrapping in ARLEQUIN. We tested overall genetic structure of 


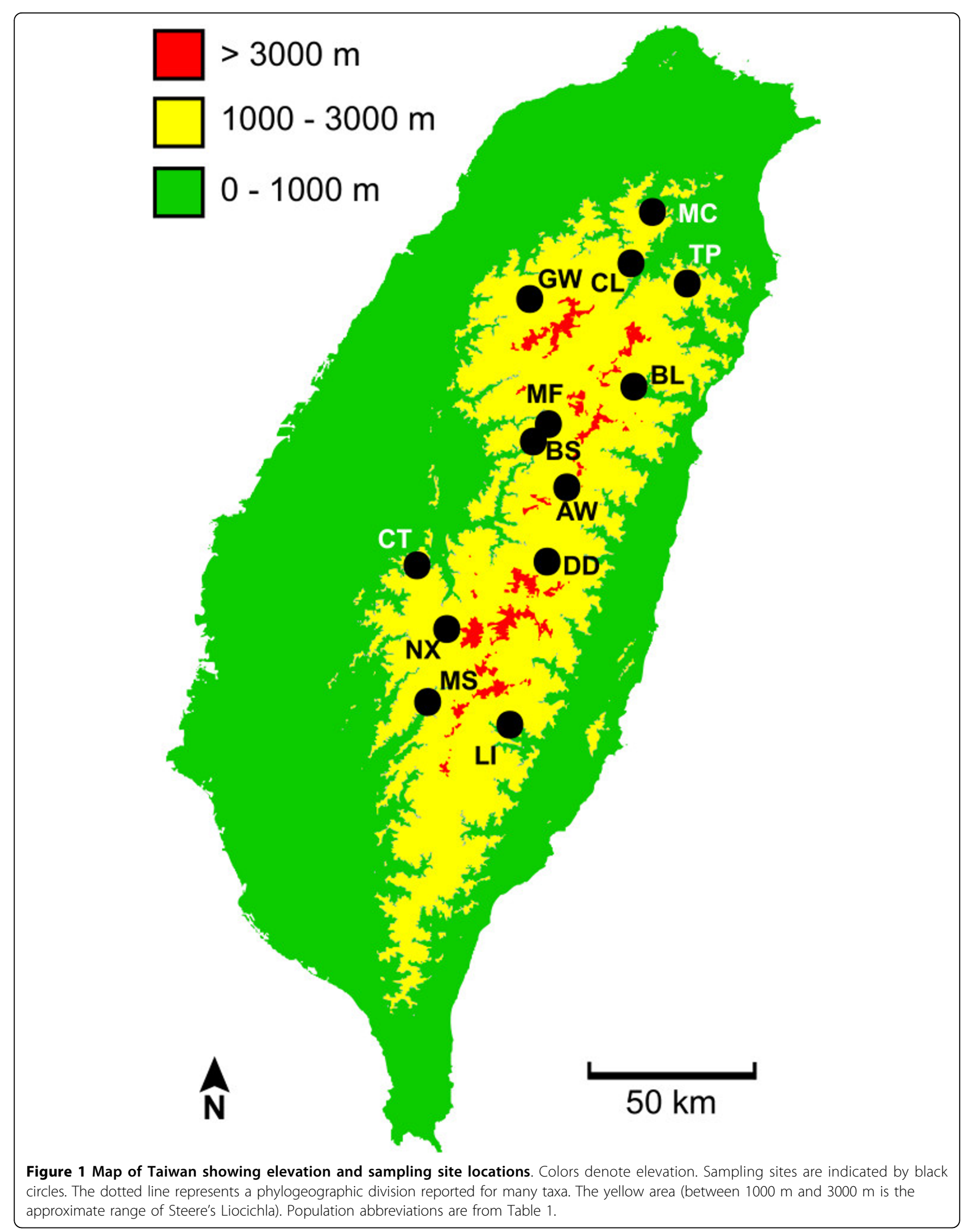


Table 1 Intrapopulation statistics for each Steere's Liocichla population separately and for all samples combined

\begin{tabular}{|c|c|c|c|c|c|c|c|c|c|c|c|c|c|}
\hline \multirow{2}{*}{ Population } & & & \multicolumn{5}{|c|}{ mtDNA } & \multicolumn{6}{|c|}{ microsatellites } \\
\hline & & & $n$ & $\pi$ & nh & $\mathrm{h}$ & $F_{S}$ & $\mathrm{n}$ & A & $A_{R}$ & $\mathrm{H}_{\mathrm{O}}$ & $\mathrm{H}_{\mathrm{E}}$ & $\mathrm{F}_{\mathrm{IS}}$ \\
\hline Aowanda & AW & South & 3 & 0.00260 & 3 & 1.00 & -0.34 & 4 & 4.00 & 4.29 & 0.86 & 0.73 & -0.171 \\
\hline Beidongyanshan & BS & North & 9 & 0.00184 & 4 & 0.69 & 0.27 & 11 & 5.43 & 4.00 & 0.80 & 0.72 & -0.121 \\
\hline Bilu & $B L$ & North & 7 & 0.00446 & 7 & 1.00 & $-3.23^{*}$ & 11 & 6.14 & 4.42 & 0.70 & 0.80 & 0.119 \\
\hline Chilan & $\mathrm{CL}$ & North & 3 & 0.00195 & 3 & 1.00 & -0.69 & 5 & 4.00 & 4.10 & 0.80 & 0.75 & -0.062 \\
\hline Chitou & $\mathrm{CT}$ & South & 9 & 0.00309 & 5 & 0.81 & 0.17 & 9 & 5.43 & 4.48 & 0.74 & 0.80 & 0.073 \\
\hline Danda & DD & South & 9 & 0.00309 & 6 & 0.92 & -1.03 & 12 & 6.29 & 4.26 & 0.72 & 0.77 & 0.060 \\
\hline Guanwu & GW & North & 9 & 0.00271 & 6 & 0.89 & -1.35 & 11 & 6.00 & 4.48 & 0.67 & 0.79 & 0.149 \\
\hline Liyuan & $\mathrm{LI}$ & South & 10 & 0.00143 & 5 & 0.76 & -1.32 & 13 & 7.57 & 4.81 & 0.84 & 0.82 & -0.034 \\
\hline Mingchi & $M C$ & North & 7 & 0.00912 & 6 & 0.95 & $-2.20^{*}$ & 10 & 5.43 & 4.47 & 0.85 & 0.80 & -0.070 \\
\hline Meifeng & MF & North & 10 & 0.00236 & 7 & 0.87 & $-2.67^{*}$ & 10 & 6.71 & 4.80 & 0.81 & 0.83 & 0.015 \\
\hline Moshan & MS & South & 8 & 0.00164 & 5 & 0.79 & -1.54 & 9 & 5.43 & 4.42 & 0.75 & 0.79 & 0.050 \\
\hline Nanxi & NX & South & 1 & $\mathrm{n} / \mathrm{a}$ & $\mathrm{n} / \mathrm{a}$ & $\mathrm{n} / \mathrm{a}$ & $\mathrm{n} / \mathrm{a}$ & 7 & 5.14 & 4.39 & 0.60 & 0.80 & 0.245 \\
\hline Taipingshan & TP & North & 6 & 0.00286 & 5 & 0.93 & -1.33 & 10 & 6.57 & 4.65 & 0.74 & 0.81 & 0.087 \\
\hline Total & & & 91 & 0.00272 & 36 & 0.90 & $-26.6^{* *}$ & 122 & 5.70 & 4.43 & 0.76 & 0.79 & 0.026 \\
\hline
\end{tabular}

The summary of genetic diversity includes sample size $(n)$ nucleotide diversity $(\pi)$, number of haplotypes (nh), haplotype diversity (h), Fu's $F_{S}\left(F_{S}\right)$, average number of alleles per locus $(A)$, allelic richness $\left(A_{R}\right)$, observed heterozygosity $\left(H_{O}\right)$, expected heterozygosity $\left(H_{E}\right)$, and inbreeding coefficient $\left(F_{I S}\right)$. Microsatellite statistics are based on the average of seven polymorphic loci.

${ }^{*} \mathrm{P}<0.05$; ${ }^{*} \mathrm{P}<0.001$

populations with a hierarchical analysis of molecular variance (AMOVA; [27]) in ARLEQUIN. To test for restricted gene flow between the north and south groups identified by previous studies, we assigned populations to either a "north" or "south" group. Based on the study of Hsu et al. [10], we used the Choushui River as the boundary between groups (see Table 1). To visualize haplotype relationships, we constructed a haplotype network using the parsimony-based algorithm of Templeton et al. [28] and implemented in the program TCS 1.21 [29]. Networks are generally better than bifurcating trees at representing the relationships of intraspecific phylogenies [30].

Microsatellite genotypes were tested for departures from Hardy-Weinberg equilibrium and evidence for linkage disequilibrium using ARLEQUIN. To assess allelic variation within populations, we calculated allele frequencies, observed (Ho) and expected (He) heterozygosities using GENEPOP 4.0.9 [31]. To correct for sample size differences, we calculated allelic richness using rarefaction with the program FSTAT 2.9.3 [32,33]. A sequential Bonferroni correction was applied to correct for multiple comparisons [34]. Principal component analysis (PCA) was applied to allele frequency data as a measure of strong multilocus associations among populations. We performed a PCA with arcsinsquare root transformed allelic frequencies using SAS (SAS Institute).

To assess genetic structure between populations, we computed pairwise $F_{S T}$ and $R_{S T}$ values for all populations. $R_{S T}$ is analogous to $F_{S T}$ but incorporates a stepwise mutation model of evolution that was specifically designed for microsatellite data [35]. Next, we performed another hierarchical AMOVA to determine the proportions of microsatellite variation among and within populations. Groups were defined in the same way as for the mtDNA AMOVA. Fixation indices and AMOVA were computed in ARLEQUIN. We also inferred genetic population structure using a Bayesian assignment approach implemented in the program BAPS 5.2 $[36,37]$. BAPS has been shown to perform better than STRUCTURE when $F_{S T}$ is small [38]. We began with a mixture analysis to determine the most probable number of populations $(K)$ given the data. We performed the "clustering of groups of individuals" analysis, considering clusters to contain a minimum of three individuals. We estimated the number and composition of clusters considering upper limits of $K$ at 2-10,15, and 20. We ran 10 repetitions for each maximum $K$. Using the groups identified in the mixture analysis, we conducted an admixture analysis with 100 realizations from the posterior of the allele frequencies. We repeated the admixture five times to confirm consistent results. We also performed a "spatial clustering of groups" analysis that incorporates geographical information in the estimation of the genetic population structure [37]. This approach provides more statistical power to infer structure when molecular data are weak. The spatial mixture analysis followed the above procedure.

Finally, to test for an association between genetic distance $\left(R_{S T}\right)$ and geographic distance, we performed a Mantel test using GenAlEx 6 [39]. We ran two Mantel tests with different geographic distances. The first used "straight-line" distances, which were simply the shortest 
distances between populations. However, Steere's Liocichla has a restricted altitudinal range and is therefore unlikely to traverse very high or very low altitudes. Thus, the second Mantel test used "least-cost" distances, which were calculated as the shortest distances between populations assuming travel was limited to elevations between $1000 \mathrm{~m}$ and $3000 \mathrm{~m}$. Straight-line and leastcost geographic distances were calculated in ArcGIS 9 (ESRI).

\section{Ecological niche modeling}

We used ecological niche modeling (ENM) to evaluate our molecular analyses of demographic history and population structure. Briefly, the general ENM approach applied here generates a map of the expected distribution of a species using data on the environmental conditions where it is known to occur and randomly selected background locations in the study area [40]. We used Maxent version 3.2 [41] to model the potential geographic distribution of Steere's Liocichla. Maxent is a general approach for characterizing probability distributions from incomplete information [41]. Maxent computes a probability distribution that describes the relative suitability of each grid cell as a function of the environmental variables at all the known occurrence locations, and when projected into geographic space it produces a map of the species' potential geographic distribution.

To construct the model, we used 19 bioclimatic variables from the WorldClim dataset with 2.5-minute spatial resolution [42] and 286 georeferenced breedingseason localities for Steere's Liocichla. The climatic variables are derived from weather station data and quantify annual variation in temperature and recipitation. Occurrence records within the same map pixel were removed to avoid pseodoreplication. To calibrate the model, we used quadratic features, and default parameters for the number of background pixels, regularization, the convergence threshold, and the maximum number of iterations. We randomly selected $75 \%$ of the occurrence locations to construct the model; the remaining $25 \%$ were set aside to test the model. We calculated the area under the receiving operator characteristic (AUC) to test whether the model could discriminate between the test localities and 10,000 localities randomly selected from across Taiwan. Finally, we selected logistic output, which ranges from 0-1 and quantify the probability of suitable environmental conditions for the species in each grid cell.

To estimate the geographic distribution and extent of suitable climate for Steere's Liocichla at the LGM (21,000 years before present), we applied the contemporary ecological niche model to a set 19 bioclimatic variables generated from CCSM3 paleoclimatic model.
In doing so, we used the fade-by-clamp option in Maxent version 3.2 to remove heavily clamped pixels from the final prediction. Failing to remove such pixels from the region might lead to erroneously extensive predictions of suitable climate when transferring a model to a different time period.

Distribution-based niche modeling makes at least three important biological assumptions. First, it assumes that a species distribution is at equilibrium with the present-day climate conditions. Second, it assumes that the distribution of the species is not strongly influence by biotic factors (e.g. competitors or predators). Third, it assumes that the climatic tolerances of the species are conserved over the timescale for which its distribution is being modeled. If any of these conditions is violated, the extent of climatically suitable habitat could be underestimated.

\section{Results}

mtDNA

A total of $1026 \mathrm{bp}$ was obtained from the mitochondrial ND2 gene. There were 48 polymorphic and 20 parsimony informative sites resulting in 36 unique haplotypes. Nuclear copies of mitochondrial genes are known in birds [43]. However, the large number of observed haplotypes and an absence of nonsensical stop-codons support a mitochondrial origin for our sequences [44]. Overall nucleotide diversity $(\pi)$ was 0.00272 for all samples combined and ranged from 0.00143 to 0.00446 (Table 1). Overall haplotype diversity was 0.9 and ranged from 0.69 to 1.00 (Table 1). AMOVA indicated non-significant differentiation (1\%) between north and south groups and significant population differentiation, with 4.5\% ( $\mathrm{P}<0.001)$ of the molecular variance partitioned among populations (Table 2). The mtDNA mismatch distribution was distinctly unimodel and did not differ significantly from a model of sudden expansion (Fig. 2). The estimate of $\tau$ was 2.9 (95\% CI 0.7-5.5). Using an avian ND2 mutation rate of $5.4 \times 10^{-8}$ [45] and assuming a generation time of two years, the time of expansion was estimated to have occurred approximately 26000 (95\% CI 6300-49700) ybp. The haplotype network showed no obvious genetic structure (Fig. 3).

\section{Microsatellites}

The microsatellite dataset showed no statistically significant deviations from Hardy-Weinberg equilibrium or evidence of lineage disequilibrium. There were a total of 137 alleles, and the average number of alleles per loci was 19.6. The number of alleles per locus ranged from 9 in lsgata21 to 51 in lsgata17. Allelic richness ranged from 4.00 in Aowanda to 7.57 in Liyuan (Table 1). PCA resulted in relatively little explanatory power with only $15.6 \%$ and $12.6 \%$ of the variation explained by the first and second 
Table 2 Analyses of molecular variance (AMOVAs) showing the distribution of genetic variation among populations of Steere's Liocichla on Taiwan

\begin{tabular}{|c|c|c|c|c|c|c|c|c|}
\hline \multirow[t]{2}{*}{ Source of variation } & \multicolumn{4}{|c|}{ mtDNA } & \multicolumn{4}{|c|}{ microsatellites } \\
\hline & df & $\%$ total & F-statistic & $P$-value & df & $\%$ total & F-statistic & $P$-value \\
\hline Among groups (north v. south) & 1 & 0.98 & 0.010 & 0.140 & 1 & -0.20 & -0.002 & 0.713 \\
\hline Among populations Within groups & 11 & 4.48 & 0.045 & 0.011 & 11 & 1.92 & 0.019 & $<0.001$ \\
\hline Within populations & 78 & 94.54 & 0.055 & 0.003 & 231 & 98.28 & 0.017 & $<0.001$ \\
\hline
\end{tabular}

principal components, respectively. It took seven principal components to explain $75 \%$ of the variation. This suggests weak multilocus associations among populations.

Overall, our analyses of population structure yielded conflicting results with different populations highlighted by different analyses. $F_{S T}$ pairwise population comparisons resulted in significant differences between the Beidongyanshan population and every other population (Table 3). $R_{S T}$ pairwise indices were larger that $F_{S T}$ comparisons (Table 3 ). This is expected because $F_{S T}$ estimated from highly variable markers is known to bias estimates downwards. This is because the proportion of the total variation among populations can never be very high when there is a lot of variation within populations [46]. $R_{S T}$ comparisons did not highlight the Beidongyanshan population as particularly distinct. Rather, there were several significant comparisons that seem haphazard with respect to geography (Table 3). AMOVA of microsatellite alleles indicated nonsignificant amounts of differentiation (0\%) distributed between north and south groups and little but significant population differentiation with $1.9 \%(\mathrm{P}<0.001)$ of the variance distributed among populations (Table 2).

The mixture analysis in BAPS indicated the presence of two distinct groups of populations, with the Meifeng population the sole member of one group and all other populations comprising the other group. The admixture

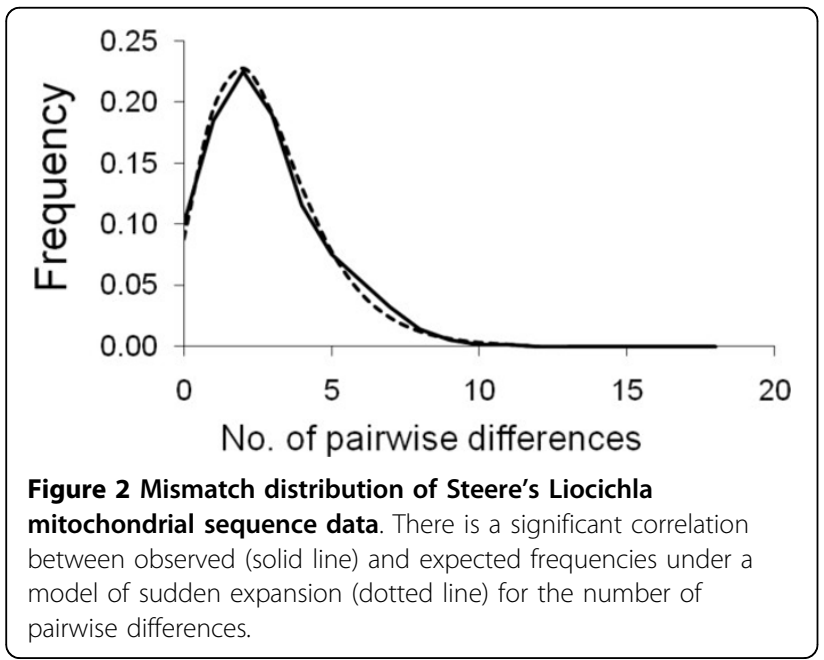

analysis assigned almost every individual to the correct group (Fig. 4). In contrast, the spatial analysis that incorporated geographic information about populations suggested only a single group. The Mantel test indicated a positive but non-significant correlation between genetic $\left(R_{S T}\right)$ and geographic distances $(\mathrm{km})$ when straight-line geographic distances were used $\left(R^{2}=0.01\right.$; $P=0.23)$. However, when least-cost geographic distances were considered, the Mantel test resulted in a positive and significant correlation $\left(R^{2}=0.07 ; P=0.04\right)$. This suggests that dispersal for these birds is, at least somewhat, limited by altitude.

\section{Ecological niche modeling}

The predicted geographic distribution of Steere's Liocichla for the present-day and LGM are shown in Fig. 5. The area under the receiving operator characteristic demonstrates that the model discriminated strongly between randomly selected locations across Taiwan, and the training (AUC $=0.93$ ) and test localities (AUC $=0.97)$.

Intriguingly, despite the many assumptions that are made when constructing distributionbased niche models, both the contemporary and LGM predictions are consistent with the findings of the molecular analyses. The model predicts that populations of Steere's Liocichla are connected by climatically suitable habitats (Fig. 5A). This result corroborates the analyses of population structure, which suggest that the degree of gene exchange is related to the geographic distance separating populations and not strong barriers to dispersal. In addition, both the geographic extent and relative suitability of habitats are predicted to have been reduced at the LGM in comparison to the present (Fig. 5B). The latter result parallels the demographic analyses, which suggest that populations may have increased in size in response to geographic expansion of suitable habitats across Taiwan.

\section{Discussion}

\section{Evolutionary history}

While we found comparatively high levels of genetic variation within populations of Steere's Liocichla, these results demonstrate a lack of genetic differentiation among populations. This is in contrast to the deep genetic divisions reported for other montane species in 


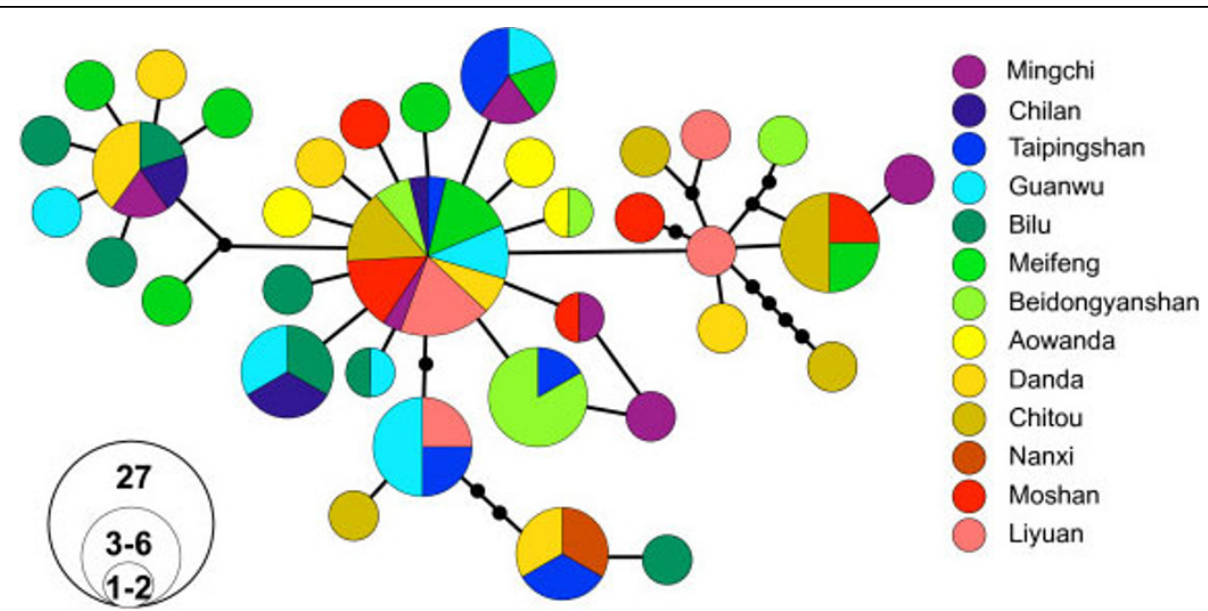

Figure 3 Mitochondrial DNA haplotype network for Steere's Liocichla ND2 sequence data. Colors indicate the population of origin. Populations from the northern group are represented by cool colors (blues and greens), and populations from the southern group are represented by warm colors (reds and yellows). Each circle represents a haplotype, and the size of the circles is proportional to its frequency. Small black circles represent unsampled haplotypes.

Taiwan. The haplotype network showed no obvious structure with respect to geography. The AMOVA indicated no genetic differentiation in either mtDNA or microsatellites between north and south groups. However, significant amounts of differentiation were partitioned among populations, implying that populations are not entirely panmictic and that dispersal among populations is likely constrained. This was true of both mtDNA and microsatellites, though the percent of variation among populations was higher for mtDNA. This is expected because mtDNA has, on average, a four times more rapid time to coalescence than nuclear loci $[47,48]$. Mantel tests were significant when least-cost distance was compared with genetic distance. This suggests that mountains, while not strict barriers to gene flow, do channel gene flow through a certain altitudinal range.

A lack of genetic structure has been found in other mountain species in Taiwan (e.g. White-bellied Rat Niviventer culturatus, [49] and Taiwan Alpine Skink Sphenomorphus taiwanensis, [50]). This might result from a recent colonization of Taiwan or historical gene flow between populations. We favor a scenario of historical gene flow for Steere's Liocichla, because it is an endemic species that's purported closest relative in Mainland China (L. omeiensis; [51]) was estimated to have diverged about 5 million years ago [52]). This suggests that either Steere's Liocichla has existed on Taiwan for a long time or that it has recently gone extinct elsewhere. The idea that populations of Steere's Liocichla

Table 3 Pairwise estimates of $\boldsymbol{F}_{S T}$ (below diagonal) and $\boldsymbol{R}_{S T}$ (above diagonal) among 13 populations of Steere's Liocichla

\begin{tabular}{|c|c|c|c|c|c|c|c|c|c|c|c|c|c|}
\hline & AW & BS & $B L$ & $\mathrm{CL}$ & CT & DD & GW & LI & MC & MF & MS & NX & TP \\
\hline AW & & 0.063 & 0.000 & 0.028 & 0.325 & 0.018 & 0.135 & 0.363 & 0.000 & 0.251 & 0.175 & 0.210 & 0.081 \\
\hline BS & 0.062 & & 0.039 & 0.000 & 0.202 & 0.000 & 0.047 & 0.265 & 0.045 & 0.246 & 0.050 & 0.129 & 0.000 \\
\hline$B L$ & 0.029 & 0.044 & & 0.041 & 0.288 & 0.055 & 0.124 & 0.370 & 0.000 & 0.301 & 0.194 & 0.219 & 0.076 \\
\hline $\mathrm{CL}$ & 0.051 & 0.097 & 0.013 & 3 & 0.057 & 0.000 & 0.000 & 0.157 & 0.000 & 0.173 & 0.018 & 0.024 & 0.000 \\
\hline CT & 0.050 & 0.043 & 0.016 & 0.004 & & 0.063 & 0.000 & 0.000 & 0.222 & 0.181 & 0.005 & 0.000 & 0.034 \\
\hline DD & 0.000 & 0.036 & 0.006 & 0.018 & 0.027 & & 0.000 & 0.159 & 0.000 & 0.240 & 0.042 & 0.010 & 0.000 \\
\hline GW & 0.000 & 0.049 & 0.017 & 0.036 & 0.000 & 0.008 & & 0.096 & 0.049 & 0.213 & 0.012 & 0.000 & 0.000 \\
\hline $\mathrm{LI}$ & 0.035 & 0.049 & 0.019 & 0.018 & 0.000 & 0.006 & 0.001 & & 0.280 & 0.194 & 0.053 & 0.018 & 0.121 \\
\hline$M C$ & 0.031 & 0.043 & 0.019 & 0.001 & 0.015 & 0.000 & 0.025 & 0.020 & & 0.267 & 0.076 & 0.121 & 0.021 \\
\hline MF & 0.050 & 0.055 & 0.000 & 0.008 & 0.006 & 0.012 & 0.019 & 0.004 & 0.020 & & 0.193 & 0.000 & 0.184 \\
\hline MS & 0.035 & 0.046 & 0.009 & 0.020 & 0.013 & 0.020 & 0.015 & 0.011 & 0.000 & 0.017 & & 0.000 & 0.016 \\
\hline$N X$ & 0.073 & 0.131 & 0.003 & 0.000 & 0.006 & 0.027 & 0.024 & 0.013 & 0.000 & 0.002 & 0.000 & & 0.000 \\
\hline $\mathrm{TP}$ & 0.009 & 0.039 & 0.002 & 0.027 & 0.000 & 0.000 & 0.000 & 0.008 & 0.000 & 0.002 & 0.000 & 0.023 & \\
\hline
\end{tabular}

Bolding indicates a significant statistic. 

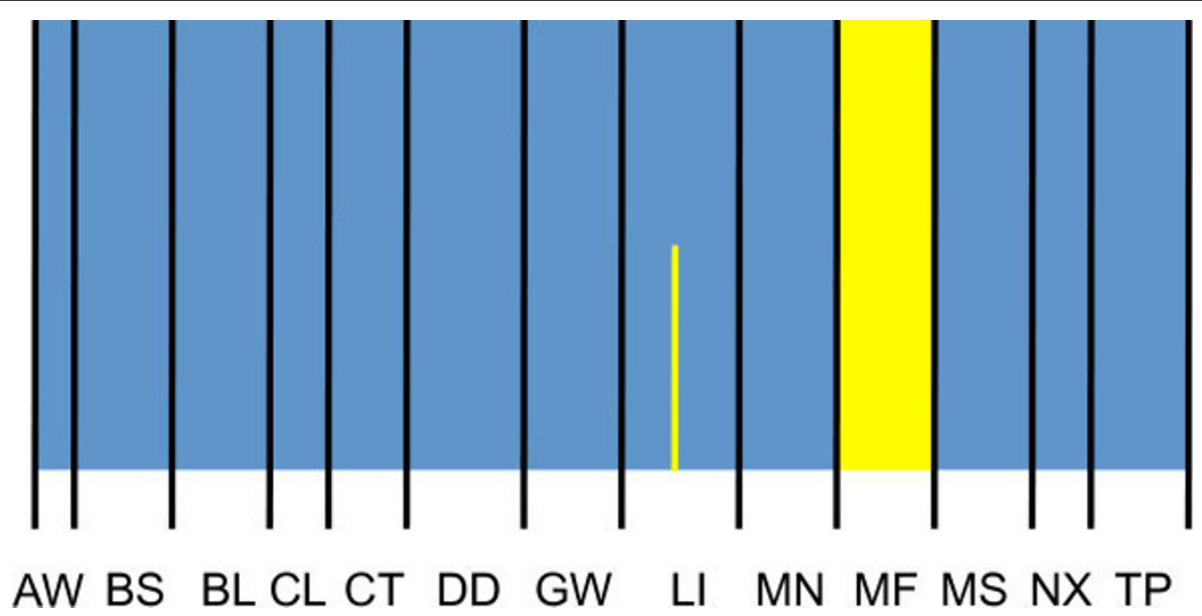

Figure 4 Admixture coefficients for Steere's Liocichla estimated using BAPS. Vertical columns correspond to individuals; black lines separate populations. Two groups were identified and they are indicated by different colors. Columns are colored in proportion to the estimated admixture coefficients for each individual. Population abbreviations are from Table 1.

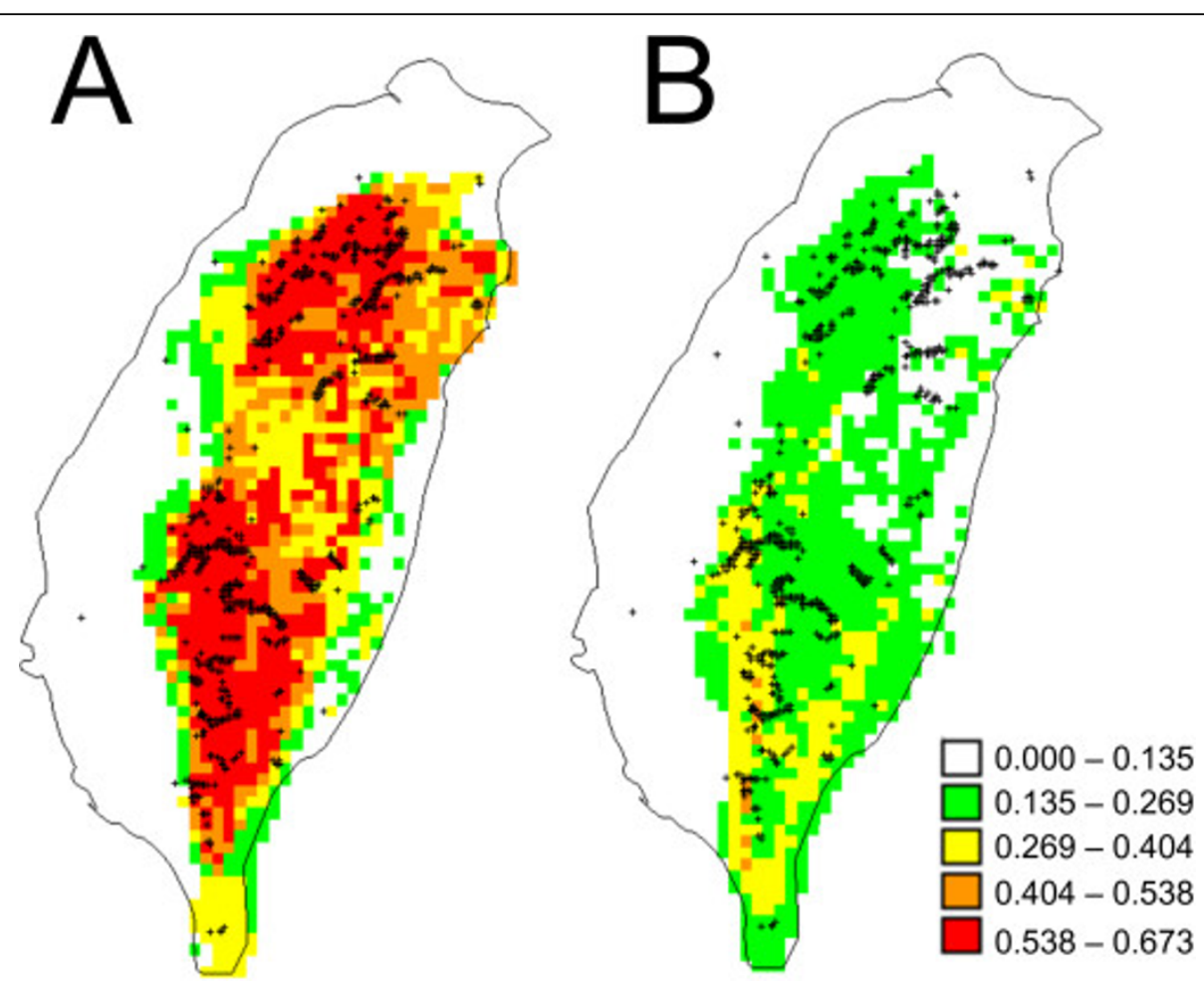

Figure 5 Predicted geographic distribution of Steere's Liocichla based on (a) present-day climatic conditions and (b) climatic conditions at the Last Glacial Maximum. Grid cells are classified by predicted suitability with white being least suitable and red being most suitable. Georeferenced occurrence records used to generate the models are indicated by plus signs.

have been historically well-connected by gene flow seems plausible given the dispersal potential of volant birds, and the geographic continuity of climatically suitable habitats over time (Fig. 5).

We also find evidence for a recent demographic expansion in Steere's Liocichla. For example, Fu's $F_{S}$, which is particularly sensitive to departures from population equilibrium [23], was negative for most populations and significantly negative when all samples were combined. The distinctly unimodal mismatch distribution did not differ significantly from a model of sudden expansion. The estimated time of this expansion was consistent with an expansion centered around the time of the Last Glacial Maximum. However, it should 
be noted that there is a large confidence interval associated with this estimate as well as assumptions involving mutation rate and generation time. Ecological niche modeling indicated that there was less suitable habitat for Steere's Liocichla during the LGM. However, marginal habitat seems to have been distributed across the island. This suggests that Steere's Liocichla occupied much of its current range during the LGM, but populations were less dense. Thus, as habitat improved with the warming climate, population densities increased leaving a pattern of recent demographic expansion. This scenario is consistent with the genetic data, which show similar levels of genetic diversity across the island rather than isolated pockets of unusually high diversity that might be interpreted as refugia. Population density increases associated with improving habitat quality following the Last Glacial Maximum might have had an underappreciated contribution to genetic patterns of expansion, especially in cases where dramatic spatial expansion from refugia seems unlikely. It would be interesting to determine whether other montane bird species in Taiwan exhibit similar patterns.

\section{Conservation implications}

Taken separately, the results of some of our analyses could be used as evidence that some populations should be of special management concern for conservation efforts. For example, we found significant differences in microsatellite $F_{S T}$ between the Beidongyanshan population and all other populations. This is surprising because there is no reason to expect restricted gene flow to or from the Beidongyanshan population; it is centrally located, only two $\mathrm{km}$ from the Meifeng population, and there is adequate habitat and a continuous population of Steere's Liocichla between Beidongyanshan and Meifeng. However, caution must be taken when interpreting $F_{S T}$ distances associated with highly polymorphic loci. Hedrick [46] has shown that relatively weak bottlenecks can cause statistically significant differences in microsatellite $F_{S T}$ distances. There is evidence that the Beidongyanshan population has recently undergone a population bottleneck and this would explain why its gene frequencies might appear to differ from the other populations.

First, the Beidongyanshan population had the lowest amount of allelic richness. Second, it had a high observed heterozygosity $\left(\mathrm{H}_{\mathrm{O}}\right)$ relative to its expected heterozygosity $\left(\mathrm{H}_{\mathrm{E}}\right)$, as indicated by the smaller than zero, and nearly significant, $F_{I S}$ value (Table 1 ). (Note that the AW population has a smaller $F_{I S}$ value, but this likely had a smaller affect on $F_{S T}$ significance because the sample size is much smaller.) A large $\mathrm{H}_{O}$ relative to $\mathrm{H}_{\mathrm{E}}$ is expected shortly after a population bottleneck because rare alleles are quickly lost, resulting in a decrease in the $\mathrm{H}_{\mathrm{E}}$. At the same time, $\mathrm{H}_{\mathrm{O}}$ levels are relatively unaffected until a new mutation-drift equilibrium can be reached because $\mathrm{H}_{\mathrm{O}}$ is mostly dictated by common alleles. Such a bottleneck may have been caused by anthropogenic disturbance from the construction of an agricultural research station at Beidongyanshan, from typhoon related disturbance, or from stochastic fluctuations in local demography. A change in $F_{S T}$ caused by a bottleneck is likely ephemeral, existing only until a new mutation-drift equilibrium is reached. Thus, individuals sampled from Beidongyanshan in the future may not show significant $F_{S T}$ differentiation from other populations. We note that in such a situation, $F_{S T}$ may reflect biologically relevant differences between populations, but this differentiation might not actually result from restricted gene flow and might be ephemeral and not of management concern. Clearly, caution should be taken before making conservation recommendations based on the results of $F_{S T}$ alone.

Because it considers information on the length of alleles, $R_{S T}$ is generally thought to perform better than $F_{S T}$ with microsatellite data, especially when there are many alleles. In contrast to $F_{S T}$ results, $R_{S T}$ comparisons did not highlight the Beidongyanshan population as particularly distinct. Instead, the few significant values of $R_{S T}$ do not seem to correlate with geography. For example, there is a significant $R_{S T}$ value between Meifeng and Beidongyanshan, which again are only two $\mathrm{km}$ apart, but no significant differences between Meifeng and Moshan or Chilan, which are much farther apart. Overall, significant $R_{S T}$ values seem haphazard with respect to geography. Again, the statistical power associated with highly variable markers, such as microsatellites, is high, so statistically significant differences should not always be taken to mean biologically significant differences [46]. It should also be noted that statically nonrandom patterns may result from the stochastic nature of population structure [53].

Bayesian analysis of population structure using BAPS found that including the Meifeng population in the pool of remaining populations decreased the fit of a panmictic total, and thus two groups were identified. Again, this is surprising because Meifeng is only two $\mathrm{km}$ from Beidongyanshan and there is adequate habitat and a continuous population of Steere's Liocichla between these two populations. However, the spatial analysis in BAPS, which incorporates information about the geographic location of populations, suggested that all populations represented one group. If separating the Meifeng population in the original BAPS analysis was a borderline case, it is understandable that the spatial model suggests only a single population (J. Corander pers. comm.). This was probably caused by an excess of private alleles in the Meifeng population. Possibly as the result of 
sampling error, Meifeng had an inordinate number of private alleles and all sampled Meifeng individuals had at least one private allele. This would explain why the admixture analysis was able to accurately assign Meifeng individuals to population. Some authors have suggested private alleles are important for conservation. For example, Funk et al. [54] defined subspecies for conservation and included the criteria that they contain "unique alleles or haplotypes." We question the practicality of this particular criterion when deciding conservation priority or making taxonomic decisions because with highly polymorphic loci, most, if not all, populations are likely to contain at least some unique alleles. In our study, all 13 populations we sampled had unique alleles. Overall, some of our analyses might seem to indicate biologically relevant differentiation of a population. However, different analyses disagree about which populations are distinct and no analysis strongly supported the distinctiveness of any population. Therefore, based on these data, none of our sampled populations should have higher conservation priority than any other.

\section{Conclusions}

We did not find the deep genetic divisions reported in other montane species in Taiwan. Instead, populations of Steere's Liocichla have been historically connected by gene flow, though inter-population migration appears largely confined to middle elevations. We found evidence of a demographic expansion that can be dated to the Last Glacial Maximum. Genetic diversity was high across the geographic range, indicating expansion was range-wide, rather than expansion from isolated refugia. Ecological niche modeling corroborated genetic data and suggested that habitat for Steere's Liocichla may have been of poorer quality during the Last Glacial Maximum. Both sources of data are consistent with a single historical scenario-Steere's Liocichla was at lower density during the Last Glacial Maximum and has subsequently expanded in population density. We suggest that such a range-wide density expansion might be an overlooked cause for the genetic patterns of demographic expansion that are regularly reported. We find significant differences among some populations in $F_{S T}$ indices and an admixture analysis. Though both of these results are often used to suggest conservation action, we affirm that statistically significant results are not necessarily biologically meaningful.

\section{Acknowledgements}

B. Zink, H. Vazquez, C.-M. Huang, and three anonymous reviewers made significant improvements to the manuscript. J. Corander assisted with the BAPS program. Fu-Hsiung Hsu and Pei-Fen Lee provided occurrence data. The Taiwan Endemic Species Research Institute helped collect samples. Work was supported under NSF-OISE 0307421 (HLM) and an NSF EAPSI fellowship to Taiwan (BDM). This work was performed in compliance of the laws and regulations of Taiwan and the United States. Birds were netted in Taiwan under permit number 090133350, and imported into the United States under USDA import permit number 49226.

\section{Author details}

${ }^{1}$ Bell Museum of Natural History, University of Minnesota, St Paul, MN 55108, USA. ${ }^{2}$ Department of Ecology, Evolution and Behavior, University of Minnesota, St Paul, MN 55108, USA. ${ }^{3}$ Geier Collections and Research Building, Cincinnati Museum Center, Cincinnati, OH 45203, USA. ${ }^{4}$ School of Forestry and Resource Conservation, National Taiwan University, Taipei, Taiwan. ${ }^{5}$ Department of Fisheries, Wildlife \& Conservation Biology, University of Minnesota, St Paul, MN 55108, USA. ${ }^{6}$ Endemic Species Research Institute, Council of Agriculture, Jiji, Taiwan. ${ }^{7}$ Department of Life Sciences, National Cheng Kung University, Tainan, Taiwan.

\section{Authors' contributions}

BM performed laboratory work, carried out analyses, and drafted the manuscript. HM conceived of the study, collected samples, and participated in the design and coordination of the study. YP collected samples, performed laboratory work, and carried out analyses. KK performed ecological niche modeling analyses and helped draft the manuscript. CY collected samples and participated in the coordination of the study. HY participated in the design and coordination of the study. All authors read and approved the final manuscript

Received: 21 October 2009 Accepted: 10 March 2010 Published: 10 March 2010

\section{References}

1. Hewitt G: The genetic legacy of the Quaternary ice ages. Nature 2000, 405:907-913.

2. Hewitt GM: Genetic consequences of climatic oscillations in the Quaternary. Philos Trans R Soc Lond B Biol Sci 2004, 359:183-195.

3. Moritz C, Patton JL, Schneider CJ, Smith TB: Diversification of rainforest faunas: an integrated molecular approach. Annu Rev Ecol Syst 2000, 31:533-563.

4. Sibuet J-C, Hsu S-K: Geodynamics of the Taiwan arc-arc collision. Tectonophysics 1997, 274:221-251.

5. Sibuet J-C, Hsu S-K: How was Taiwan created? Tectonophysics 2004, 379:159-181

6. Voris HK: Maps of Pleistocene sea levels in Southeast Asia: shorelines, river systems and time durations. J Biogeogr 2000, 27:1153-1167.

7. Shih $\mathrm{H}-\mathrm{T}$, Hung $\mathrm{H}-\mathrm{C}$, Schubart $\mathrm{CD}$, Chen $\mathrm{CA}$, Chang H-W: Intraspecific genetic diversity of the endemic freshwater crab Candidiopotamon rathbunae (Decapoda, Brachyura, Potamidae) reflects five million years of the geological history of Taiwan. J Biogeogr 2006, 33:980-989.

8. Toda M, Nishida M, Matsui M, Lue K-Y, Ota H: Genetic Variation in the Indian Rice Frog, Rana limnocharis (Amphibia: Anura), in Taiwan, as Revealed by Allozyme Data. Herpetologica 1998, 54:73-82.

9. Tsai C-Y: Population phylogeny of Gekko hokouensis based on mitochondrial 12S rRNA and cytochrome b sequences. Master's thesis National Sun Yat-sen University 1999.

10. Hsu F-H, Lin F-J, Lin Y-S: Phylogeographic structure of the Formosan wood mouse, Apodemus semotus Thomas. Zool Stud 2001, 40:91-102.

11. Chen H-C: Preliminary study on mitochondrial DNA sequence and population variation of Rana sauteri. Master's thesis National Taiwan University 1994.

12. Wu M-C: Phylogeography variation of Siberian weasel (Mustela sibirica) in Taiwan, based on control region sequences of mitochondrial DNA. Master's thesis National Sun Yat-sen University 2004.

13. Zink RM, Blackwell-Rago RC, Ronquist F: The shifting roles of dispersal and vicariance in biogeography. Proc Biol Sci 2000, 267:497-503.

14. Hsu Y-T: The evolutionary relationship of Chinese bulbul (Pycnonotus sinensis) and Taiwan bulbul (P. taivanus) revealed by their population genetic structure. Master's thesis National Taiwan University 1999.

15. Chen H-C, Yuan H-W, Lin Y-S: Genetic diversity and population genetic structure of the Ring-necked Pheasant (Phasianus colchicus) in Taiwan. Journal of the Experimental Forest of National Taiwan University 2004, 18:65-75. 
16. Huang $Y-K$ : The island effect on the avifauna of the mountain islands in southern Taiwan. Master's thesis National Pingtung University of Science and Technology 2004.

17. Tsai Y-L: Geographic song variation of Steere's Liocichla (Liocichla steerii). Master's thesis National Taiwan University 2005.

18. Seutin G, White BN, Boag PT: Preservation of Avian Blood and Tissue Samples for DNA Analyses. Canadian Journal of Zoology-Revue Canadienne De Zoologie 1991, 69:82-90.

19. Sorenson MD, Ast JC, Dimcheff DE, Yuri T, Mindell DP: Primers for a PCRBased Approach to Mitochondrial Genome Sequencing in Birds and Other Vertebrates. Mol Phylogen Evol 1999, 12:105-114.

20. Yeung C, Huang YJ, Li SH: Development of polymorphic microsatellite markers for the Steere's Liocichla (Liocichla steerii). Mol Ecol Notes 2004 4:420-422.

21. Huang YJ, Chen CS, Li SH: Polymorphic tetranucleotide microsatellite loci in the Hwamei (Garrulax canorus canorus) (Timaliidae). Mol Ecol Notes 2004, 4:170-172.

22. Rozas J, Sanchez-DelBarrio JC, Messeguer X, Rozas R: DnaSP, DNA polymorphism analyses by the coalescent and other methods. Bioinformatics 2003, 19:2496-2497.

23. Fu YX: Statistical tests of neutrality of mutations against population growth, hitchhiking and background selection. Genetics 1997, 147:915-925.

24. Excoffier L, Laval G, Schneider S: Arlequin (version 3.0): An integrated software package for population genetics data analysis. Evol Bioinform Online 2005, 1:47-50

25. Schneider S, Excoffier L: Estimation of demographic parameters from the distribution of pairwise differences when the mutation rates vary among sites: Application to human mitochondrial DNA. Genetics 1999, 152:1079-1089

26. Rogers AR, Harpending H: Population growth makes waves in the distribution of pairwise genetic differences. Mol Biol Evol 1992, 9:552-569.

27. Excoffier L, Smouse PE, Quattro JM: Analysis of molecular variance inferred from metric distances among DNA haplotypes: application to human mitochondrial DNA restriction data. Genetics 1992, 131:479-491.

28. Templeton AR, Crandall KA, Sing CF: A cladistic analysis of phenotypic associations with haplotypes inferred from restriction endonuclease mapping and DNA sequence data. III. Cladogram estimation. Genetics 1992, 132:619-633

29. Clement M, Posada D, Crandall KA: TCS: a computer program to estimate gene genealogies. Mol Ecol 2000, 9:1657-1659.

30. Posada D, Crandall KA: Intraspecific gene genealogies: trees grafting into networks. Trends Ecol Evol 2001, 16:37-45.

31. Rousset F: genepop'007: a complete re-implementation of the genepop software for Windows and Linux. Molecular Ecology Resources 2008 8:103-106.

32. Goudet J: FSTAT (Version 1.2): A computer program to calculate Fstatistics. J Hered 1995, 86:485-486.

33. FSTAT Version 2.9.3. a program to estimate and test gene diversities and fixation indices. [http://www2.unil.ch/popgen/softwares/fstat.htm].

34. Rice WR: Analyzing Tables of Statistical Tests. Evolution 1989, 43:223-225.

35. Slatkin M: A measure of population subdivision based on microsatellite allele frequencies. Genetics 1995, 139:457-462.

36. Corander J, Marttinen P: Bayesian identification of admixture events using multilocus molecular markers. Mol Ecol 2006, 15:2833-2843.

37. Corander J, Marttinen P, Siren J, Tang J: Enhanced Bayesian modelling in BAPS software for learning genetic structures of populations. BMC Bioinformatics 2008, 9:539.

38. Latch EK, Dharmarajan G, Glaubitz JC, Rhodes OE Jr: Relative performance of Bayesian clustering software for inferring population substructure and individual assignment at low levels of population differentiation. Conserv Genet 2006, 7:295-302.

39. Peakall R, Smouse PE: genalex 6: genetic analysis in Excel. Population genetic software for teaching and research. Mol Ecol Notes 2006, 6:288-295.

40. Kozak KH, Graham CH, Wiens JJ: Integrating GIS-based environmental data into evolutionary biology. Trends Ecol Evol 2008, 23:141-148.

41. Phillips SJ, Anderson RP, Schapire RE: Maximum entropy modeling of species geographic distributions. Ecol Model 2006, 190:231-259.
42. Hijmans RJ, Cameron SE, Parra JL, Jones $\mathrm{PG}$, Jarvis $\mathrm{A}$ : Very high resolution interpolated climate surfaces for global land areas. International Journal of Climatology 2005, 25:1965-1978.

43. Sorenson MD, Quinn TW: Numts: A challenge for avian systematics and population biology. The Auk 1998, 115:214-221.

44. Zhang D-X, Hewitt GM: Nuclear integrations: challenges for mitochondrial DNA markers. Trends Ecol Evol 1996, 11:247-251.

45. Zink RM, Pavlova A, Rohwer S, Drovetski SV: Barn swallows before barns: population histories and intercontinental colonization. Proc R Soc B 2006, 273:1245-1251.

46. Hedrick PW: Highly variable loci and their interpretation in evolution and conservation. Evolution 1999, 53:313-318.

47. Zink RM, Barrowclough GF: Mitochondrial DNA under siege in avian phylogeography. Mol Ecol 2008, 17:2107-2121.

48. Moore WS: Inferring Phylogenies from mtDNA Variation: MitochondrialGene Trees Versus Nuclear-Gene Trees. Evolution 1995, 49:718-726.

49. Hsu F-H, Lin F-J, Lin Y-S: Phylogeographic variation in mitochondrial DNA of the Formosan white-bellied rat Niviventer culturatus. Zool Stud 2000, 39:38-46.

50. Guo Q-H: The population genetic structure of Sphenomorphus taiwanensis. Master's thesis National Taiwan Normal University 2002.

51. Collar NJ, Robson C: Family Timaliidae (Babblers). Handbook of Birds of the World Vol 12 Picarthartes to Tits and Chickadees Barcelona: Lynx Edicionsdel Hoyo J, Elliott A, Christie DA 2007, 70-291.

52. Luo X, Qu Y-H, Han L-X, Li S-H, Lei F-M: A phylogenetic analysis of laughingthrushes (Timaliidae: Garrulax) and allies based mitochondrial and nuclear DNA sequences. Zool Scr 2008, 38:9-22.

53. Ball RM, Neigel JE, Avise JC: Gene genealogies within the organismal pedigrees of random mating populations. Evolution 1990, 54:1414-1422.

54. Funk WC, Mullins TD, Haig SM: Conservation genetics of snowy plovers (Charadrius alexandrinus) in the Western Hemisphere: population genetic structure and delineation of subspecies. Conserv Genet 2007, 8:1287-1309.

doi:10.1186/1471-2148-10-71

Cite this article as: McKay et al.: Recent range-wide demographic expansion in a Taiwan endemic montane bird, Steere's Liocichla (Liocichla steerii). BMC Evolutionary Biology 2010 10:71.

\section{Submit your next manuscript to BioMed Central and take full advantage of:}

- Convenient online submission

- Thorough peer review

- No space constraints or color figure charges

- Immediate publication on acceptance

- Inclusion in PubMed, CAS, Scopus and Google Scholar

- Research which is freely available for redistribution

Submit your manuscript at www.biomedcentral.com/submit
Biomed Central 